
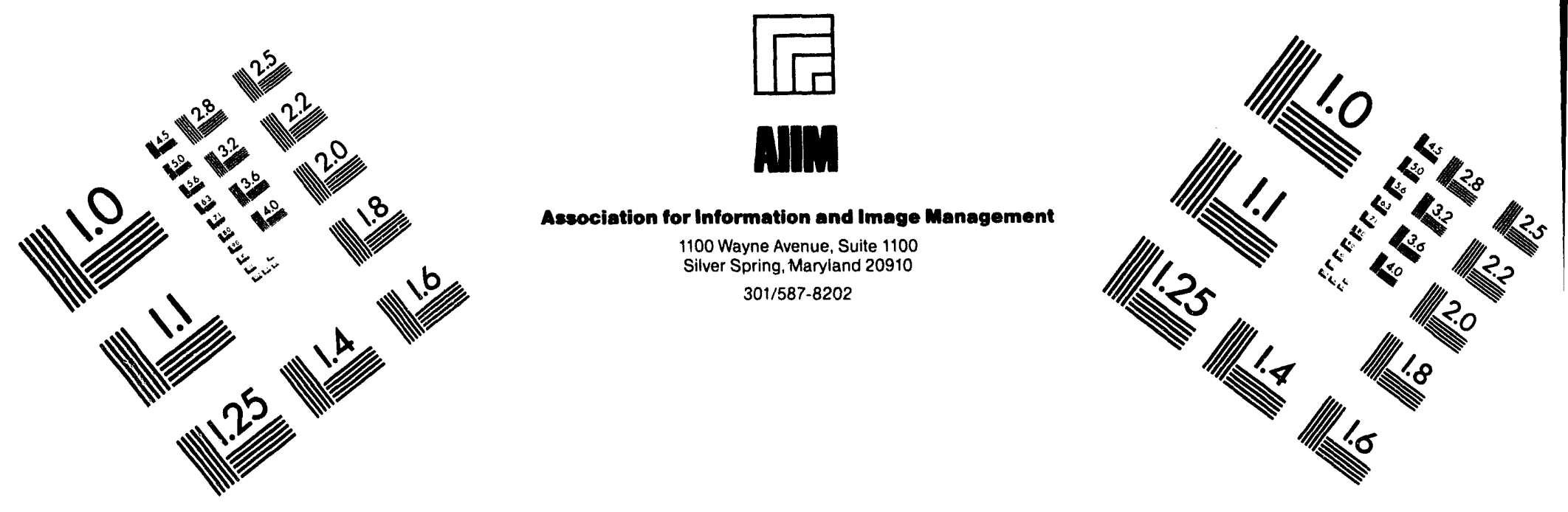

\title{
Centimeter
}

|m Inches
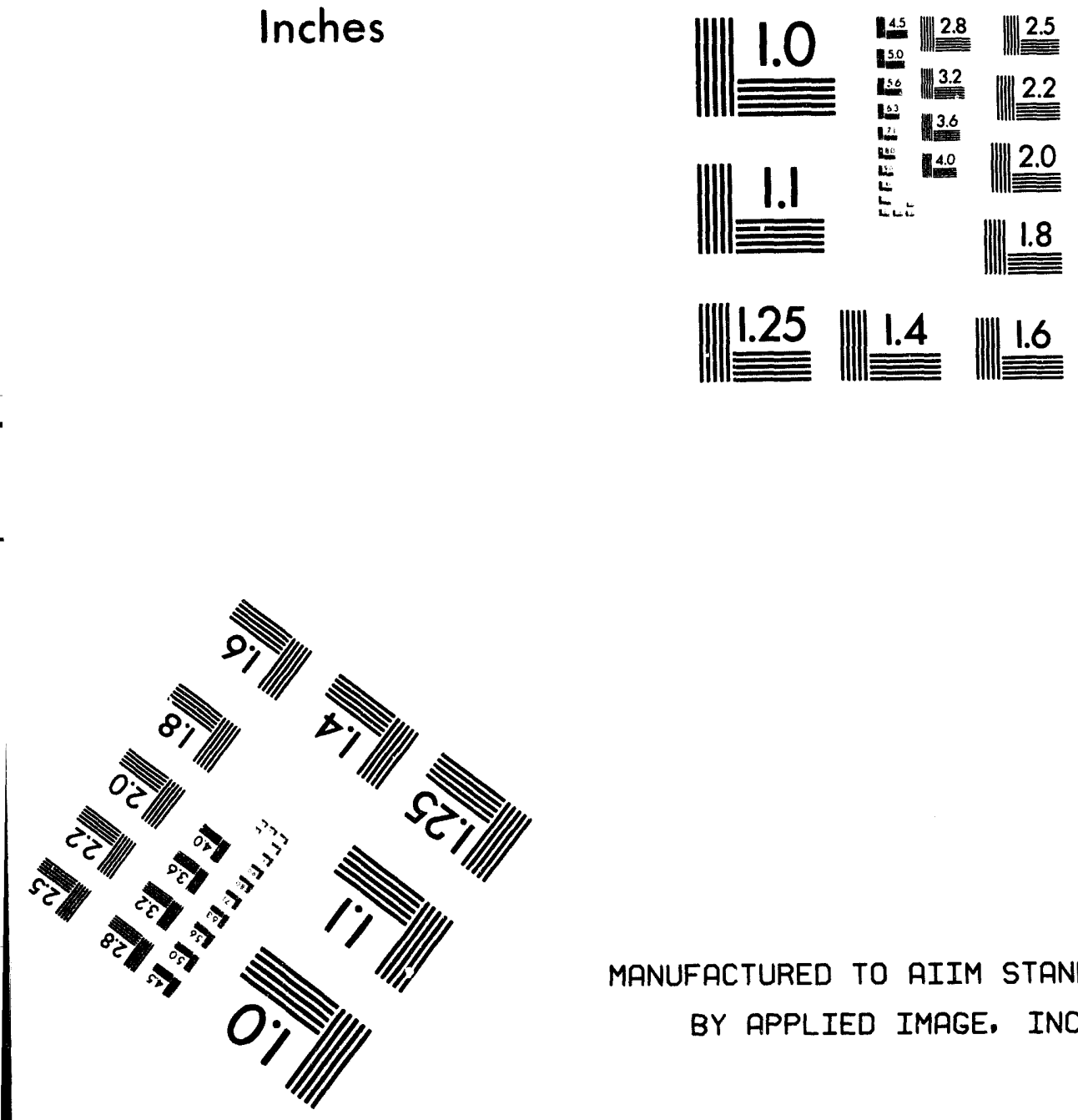

MANUFACTURED TO AIIM STANDARDS

BY APPLIED IMAGE, INC.

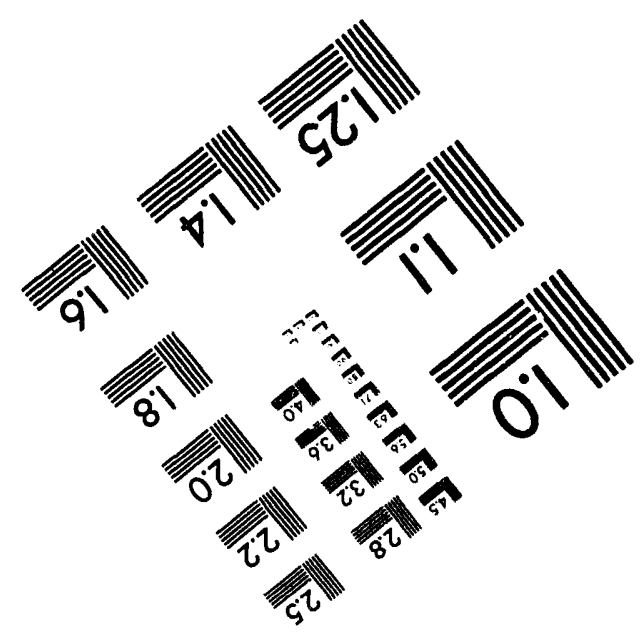



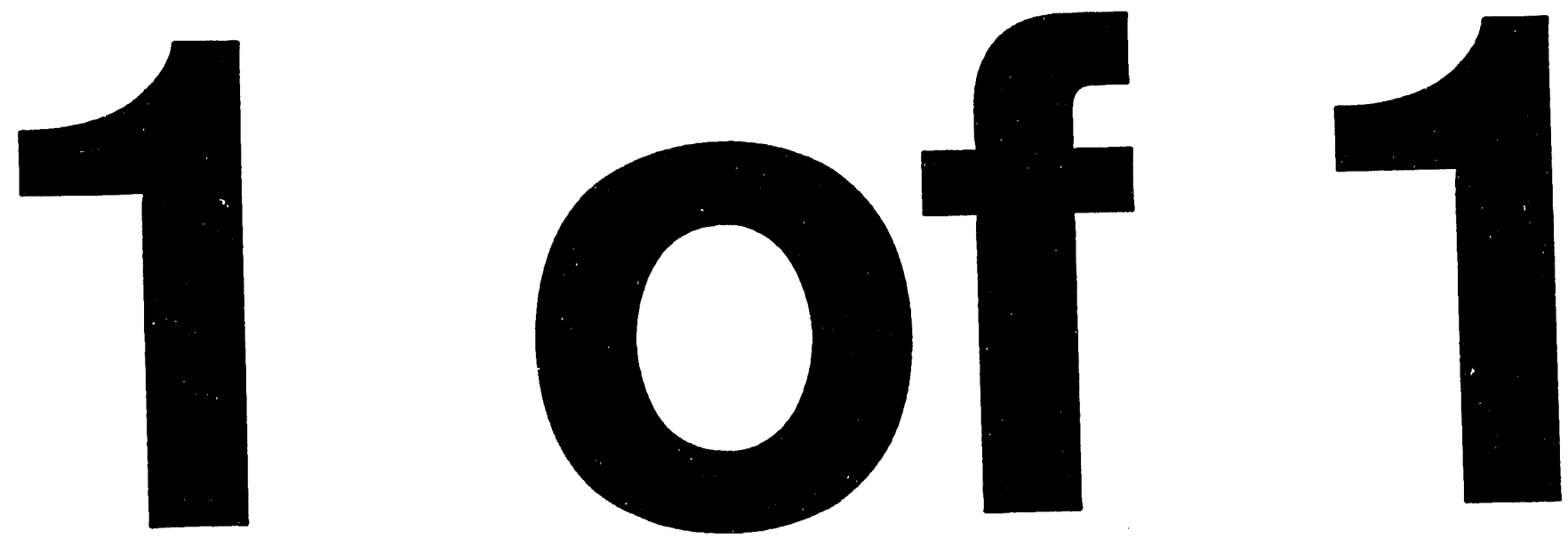
DOE/ER/40561-127-INT93-00-48

\section{A Threshold for Dissipative Fission}

\section{Michael Thoennessen}

National Superconducting Cyclotron Laboratory and Department of Physics and Astronomy, Michigan State University, East Lansing, MI 48824

and

\section{George F. Bertsch}

Department of Physics, FM-15, Institute for Nuclear Theory, HN-12, University of Washington, Seattle, WA 98195

\section{RECEIVED MAR $\rightarrow 3$ S OSTI}

\section{PREPARED FOR THE U.S. DEPARTMENT OF ENERGY UNDER GRANT DE-FG06-90ER40561}

This report was prepared as an account of work sponsored by the United States Government. Neither the United States nor any agency thereof, nor any of their employees, makes any warranty, express or implied, or assumes any legal liability or responsibility for the accuracy, completeness, or usefulness of any information, apparatus, product; or process disclosed, or represents that its use would not infringe privately owned rights. Reference herein to any specific commercial product, process, or service by trade name, mark, manufacturer, or otherwise, does not necessarily constitute or imply its endorsement, recommendation, or favoring by the United States Government or any agency thereof. The views and opinions of authors expressed herein do not necessarily state or reflect those of the United States Government or any agency thereof. 


\title{
A Threshold for Dissipative Fission
}

\author{
M. Thoennessen ${ }^{(1)}$ and G. F. Bertsch ${ }^{(2)}$ \\ (1) National Superconducting Cyclotron Laboratory and Department of Physics $\&$ \\ Astronomy Michigan State University, East Lansing, Michigan 48824 \\ (2) Department of Physics FM-15, University of Washington, Seattle WA 98105
}

(September 21, 1993)

\begin{abstract}
We examine the empirical domain of validity of statistical theory, as applied to fission data on pre-fission neutron, charged particle, and $\gamma$-ray multiplicities. Systematics are found of the threshold excitation energy for the appearance of nonstatistical fission. From the data on systems with not too high fissility, the relevant phenomenological parameter is the ratio of the threshold temperature $T_{\text {thresh }}$ to the (temperature dependent) fission barrier height $E_{B a r}(T)$. The statistical model reproduces the data for $T_{\text {thresh }} / E_{B a r}(T)<0.26 \pm 0.05$ but underpredicts the multiplicities at higher $T_{\text {thresh }} / E_{B \text { ar }}(T)$ independent of mass and fissility of the systems.
\end{abstract}

PACS 24.75. $+\mathrm{i}, 25.70 . \mathrm{Jj}$ 
It is well established that the fission process of hot nuclear systems cannot be described within the Bohr-Wheeler statistical theory, equivalent to the transition state theory of unimolecular reactions. At high excitation energies the pre-fission neutron, charged particle and giant dipole resonance (GDR) $\gamma$-ray multiplicities exceed the predictions of the statistical model calculations, although the model works well at low excitation energy. Many systems have been studied extensively [1], but this apparent hindrance or delayed onset of fission is still not well understood. Phenomenologically, the fission hindrance can be described in the framework of Kramer's model [2] as a consequence of either very large or very small dissipation of the collective motion. Thus, this data presents to the theorist the problem of understanding the dissipation and how it depends on excitation energy.

In this work we search for systematic trends of the validity of the statistical model by assembling data over a wide range of masses and fissilities. In particular, we shall tabulate the threshold energy $E_{\text {thresh }}$ marking the upper limit of energies where the statistical theory applies. Although a large set of excitation functions covering the relevant energy range exists already for quile some time, no detailed analysis of these data have been performed. In the present paper we extract and analyze the threshold energy from a variety of different measurements. We find a rather simple correlation, which we do not understand, however.

Table I lists the analyzed fissioning systems following fusion evaporation reactions. Prefission neutron, charged particle, and GDR- $\gamma$-ray multiplicity measurements are included. The first entries in the table are the reaction partners and the compound nucleus they form. Following this is the fissility of the compound system, $x_{\text {fiss. }}$. The next entry is the threshold energy, determined as follows. For data on the neutron and charged particle multiplicities, the threshold was extracted from excitation function plots of the multiplicities which compared experimental data with statistical model calculations. $E_{\text {thresh }}$ is defined as the compound nucleus excitation energy where the model starts to deviate from the data. Uncertainties were estimated from the graphs. One measurement calls for special comment, namely the reaction ${ }^{19} \mathrm{~F}+{ }^{232} \mathrm{Th} \rightarrow{ }^{251} \mathrm{Es}$. Here $\mathrm{E}_{\text {thresh }}$ is the estimated quoted in the experimental paper, Ref. [5], but since that energy is far below the fusion barrier it should 
be taken with caution. The bottom entries in the table are measurements of GDR $\gamma$ rays, which do not yet provide detailed excitation functions. The threshold energy is only quoted as a range, where the lower value corresponds to the highest measured excitation energy where the statistical model still could describe the data. The upper value was chosen to be in the middle between the lower energy and the first excitation energy where dissipation had to be included in order to fit the $\gamma$-ray spectrum.

In addition to the particle/ $\boldsymbol{\gamma}$-ray multiplicity data of fusion reactions we also analyzed the data from sequential fission of the peripheral collision of ${ }^{40} \mathrm{Ar}+{ }^{232} \mathrm{Th}$ at $30 \mathrm{MeV} / \mathrm{A}$ (Table II). The threshold energy was deduced from Figure 2 of Ref. [14]. The crossover energies where the calculated fission probabilities reach the measured fission probabilities were attributed to the onset of fission hindrance. It should be emphasized that $\mathrm{E}_{\text {thresh }}$ in this case is not a directly measured quantity, but was derived from a model calculation.

The extracted values of $E_{t h r e s h}$ of Table I and II are summarized in Figure 1 and shown as a function of the mass number of the fissioning system. The different symbols correspond to the following experimental methods: Neutron multiplicities following heavy-ion fusion (o) and proton induced fusion ( $\square$ ), charged particle multiplicities $(\bullet)$, GDR- $\gamma$-ray multiplicities $(x)$, and peripheral reactions $(\Delta)$. At low masses, $E_{\text {thresh }}$ is large $(\sim 80 \mathrm{MeV})$ and it seems to drop to lower values for heavier systems. However, the data are spread over a wide range and no clear trends can be observed.

Figure 2 shows the same data as a function of the fissility of the system. Here it is obvious that $E_{\text {thresh }}$ drops dramatically from $80 \mathrm{MeV}$ down to $20 \mathrm{MeV}$ within a rather narrow range of fissilities around 0.72. Only two data points do not follow this general trend. The GDR- $\gamma$-ray multiplicity measurements of the two ${ }^{32} \mathrm{~S}$ induced reactions show a very large threshold energy even at very large fissilities. It is difficult to see how the large $\mathrm{E}_{\text {thresh }}$ obtained in the GDR analysis can be reconciled with the smaller $\mathrm{E}_{\text {thresh }}$ obtained in particle multiplicity studies for nuclei with similar fissilities. This discrepancy can not be attributed to the different methods applied, since two other data points deduced from GDR measurements $\left({ }^{200} \mathrm{~Pb}\right.$ and $\left.{ }^{224} \mathrm{Th}\right)$ follow the general trend rather nicely. However, it might 
be useful to repeat the measurements for the same systems studied by the other technique, in order to try to resolve this disagreement.

In the following we try to find a parameterization of $E_{\text {thresh }}$ which is independent of the size of the system or the fissility. Obviously the temperature is an important parameter, and we present in Table I the threshold temperature $T_{\text {thresh }}$ corresponding to the energy threshold $E_{t h e s h}$. To compute this, the rotational energy $E_{\text {rot }}$ must be subtracted from the excitation energy. As a function of angular momentum $l$, we assume $T_{\text {thresh }}(l)=\sqrt{\left(E_{\text {thresh }}-E_{\text {rot }}(l)\right) / a}$, with the level density $a=A / 9$. We then average over angular momenta contributing to the fission reaction as

$$
T_{\text {thresh }}=\frac{\sum_{l=0}^{l_{\max }} T_{\text {thresh }}(l) \times \sigma_{\text {fiss }}(l)}{\sum_{l=0}^{l_{\max }} \sigma_{\text {fiss }}(l)} .
$$

$T_{\text {thresh }}$ is therefore the mean temperature for the systems leading to fission.

We extract the fission barrier in a similar way, using the Sierk's angular momentum dependent fission barriers [15],

$$
E_{B a r}=\frac{\sum_{l=0}^{l_{\max }} E_{B a r}(l) \times \sigma_{f i s s}(l)}{\sum_{l=0}^{l_{\max }} \sigma_{f i s s}(l)}
$$

The fission barrier may also depend on the temperature, so for completeness we also calculated the temperature-dependent barrier $E_{B a r}(T)$ using the parameterization of Ref. [16]. The values for $\mathrm{T}_{\text {thresh }}, \mathrm{E}_{B a r}$ and $\mathrm{E}_{B a r}(\mathrm{~T})$ are listed in Tables I and II.

Figure 3 shows the ratio of the threshold temperature $\mathrm{T}_{\text {thresh }}$ over the temperature dependent fission barrier $E_{B a r}(T)$ as a function of mass. With the exception of the two ${ }^{32} \mathrm{~S}$ induced reactions, this quantity seems to be independent of the mass and therefore also independent of the fissility of the system. This is also the case for the Sierk barrier without any temperature dependence.

There also exists no discrepancy between the different probes that were used to measure the threshold energy. The majority of the data points were extracted from neutron multiplicity measurements and they agree very well with experiments of charged particle and GDR $\gamma$-ray multiplicities. Also the proton induced reaction and the peripheral data are in agreement. 
If we ignore the two ${ }^{32} \mathrm{~S}$ induced reactions, we can plot the remaining data points on a linear scale (Figure 4) and extract a mean value (excluding ${ }^{251} \mathrm{Es}$ ) of $\mathrm{T}_{\text {thresh }} / \mathrm{E}_{B a r}(\mathrm{~T})=$ $0.26 \pm 0.05$, and a somewhat smaller value $0.20 \pm 0.05$ using the Sierk barrier without any temperature dependence.

The significance and interpretation of this empirical relation is not obvious. The parameter $\mathrm{T}_{\text {thresh }} / \mathrm{E}_{B a r}$ enters into a number of considerations. It must be small in order for a compound system to be formed, but this criterion is met for much larger values than we found for the threshold. The parameter explicitly enters into the prefactor of the decay formula in the limit of small dissipation and underdamped collective motion (eq. (28) of Ref. [2]). If the friction coefficient were constant, the fission rate would decrease below the statistical decay rate at higher temperature consistent with the experiments. However, it is established that the high temperature region is overdamped, which is supported by some microscopic calculations $[17,18]$ and also by experimental observations [19,1]. Thus, Kramer's underdamped solution does not apply and can not be the explanation for the extracted relation.

Although it is obvious that nuclear dissipation is temperature dependent, the origin of this dependence is still not understood. As Kramers pointed out in his original paper [2], it is far from clear whether the common assumption of a linear friction is justified [20]. Only very recently have calculations attempted to reproduce the excitation dependence of prefission evaporation multiplicities as well as fission probabilities [21]. The present observation of a systematic behavior in a wide range of measurements and the existence of a numerical parameter for the onset of dissipation effects is valuable for future theoretical calculations and has to be understood within the models.

This work was supported by the U.S. National Science Foundation under Grant No. PHY-92-14992 and the Department of Energy under grant No. DE-FG06-90ER40561. 


\section{REFERENCES}

[1] D. Hilscher and H. Rossner, Ann. Phys. Fr 17, 471 (1992).

[2] H.A. Kramers, Physica 7, 284 (1940).

[3] A. Gavron, et al., Phys. Rev. C 35, 579 (1987).

[4] D. J. Hinde, R. J. Charity, G. S. Foote, J. R. Leigh, J. O. Newton, S. Ogaza, and A. Chatterjee, Nucl. Phys. A452, 550 (1986).

[5] J. O. Newton, D. J. Hinde, R. J. Charity, J. R. Leigh, J. J. M. Bokhorst, A. Chatterjee, G. S. Foote, and S. Ogaza, Nucl. Phys. A483, 126 (1988).

[6] D. J. Hinde, D. Hilscher, and H. Rossner, Nucl. Phys. A502, 497c (1989).

[7] D. J. Hinde, D. Hilscher, H. Rossner, B. Gebauer, M. Lehmann, and M. Wilpert, Phys. Rev. C 45, 1229 (1992).

[8] M. Strecker, R. Wien, P. Plischke, and W. Scobel, Phys. Rev. C 41, 2172 (1990).

[9] J. P. Lestone, J. R. Leigh, J. O. Newton, J. X. Wei, J. X. Chen, S. Elfstrom, and D. G. Popescu, Phys. Rev. Lett. 67, 1078 (1991).

[10] R. Butsch, M. Thoennessen, D. R. Chakrabarty, M. G. Herman, and P.Paul, Phys. Rev. C 41, 1530 (1990).

[11] I. Diószegi, D. J. Hofman, C. P. Montoya, S. Schadmand, and P. Paul, Phys. Rev. C 46, 627 (1992), see discussion in Ref. [13].

[12] M. Thoennessen, D. R. Chakrabarty, M. G. Herman, R. Butsch, and P.Paul, Phys. Rev. Lett. 59, 2860 (1987).

[13] D. J. Hofman, B. B. Back, I. Diószegi, C. P. Montoya, S. Schadmand, R. Varma, and P. Paul, submitted to Phys. Rev. Lett.

[14] E.-M. Eckert, et al., Phys. Rev. Lett. 64, 2483 (1990). 
[15] A.J. Sierk, Phys. Rev. C33, 2039 (1986).

[16] J. O. Newton, D. G. Popescu, and J. R. Leigh, Phys. Rev. C42, 1772 (1990).

[17] H. Hofmann, S. Yamaji, and A. Jensen, Phys. Lett. B286 1 (1992).

[18] B. Bush, G. Bertsch, and B. Brown, Phys. Rev. C45 1709 (1992).

[19] R. Butsch, D. J. Hofman, C. P. Muntoya, P. Paul, and M. Thoennessen, Phys. Rev. C 44, 1515 (1991).

[20] M. Nemes and H. Weidenmueller, Phys. Rev. C24 944, (1981).

[21] P. Fröbrich, I. I. Gontchar, N. D. Mavlitov, HMI-preprint HMI 1992/P1-Fröb 1, Nucl. Phys, in press. 


\section{FIGURES}

FIG. 1. The extracted threshold energy $E_{\text {thresh }}$, where dissipation effects start to influence the fission process in hot nuclei as a function of mass. The different symbols correspond to neutron multiplicities following heavy-ion fusion (o) and proton induced fusion ( $(\mathbf{)})$, charged particle multiplicities $(\bullet)$, GDR- $\boldsymbol{\gamma}$-ray multiplicities $(x)$, and peripheral reactions $(\Delta)$. The data were taken from References listed in Tables 1 and 2.

FIG. 2. The extracted threshold energy $E_{t h r e s h}$, as a function of the fissility. Symbols as in Fig. 1.

FIG. 3. The ratio of the threshold temperature $T_{\text {thresh }}$ over the temperature dependent fission barrier $\mathrm{E}_{B a r}(\mathrm{~T})$ as a function of mass. Symbols as in Fig. 1.

FIG. 4. Linear presentation of the ratio of the threshold temperature $T_{\text {thresh }}$ over the temperature dependent fission barrier $\mathrm{E}_{B a r}(\mathrm{~T})$ as a function of mass. Symbols as in Fig. 1. 


\section{TABLES}

TABLE I. Reactions, compound nuclei $(C N)$, fissilities $\left(x_{f i s s}\right)$, threshold energies ( $\left.E_{t h r e s h}\right)$, threshold temperatures $\left(T_{\text {thresh }}\right)$, mean fission barriers $E_{B a r}$, temperature dependent mean fission barriers $E_{B a r}(T)$, for the analysed reactions. The last two columns list the experiment type and the references. All energies and temperatures are given in $\mathrm{MeV}$.

\begin{tabular}{|c|c|c|c|c|c|c|c|c|}
\hline Reaction & $\mathrm{CN}$ & $x_{\text {fiss }}$ & $\mathrm{E}_{\text {thresh }}$ & $\mathrm{T}_{\text {thresh }}$ & $E_{B a r}$ & $\mathrm{E}_{B a r}(\mathrm{~T})$ & & Reference \\
\hline${ }^{16} \mathrm{O}+{ }^{142} \mathrm{Nd}$ & ${ }^{158} \mathrm{Er}$ & 0.60 & $80 \pm 10$ & $1.83 \pm 0.11$ & $11.2 \pm 2.0$ & $8.1 \pm 2.0$ & $n$ & [3] \\
\hline${ }^{18} \mathrm{O}+{ }^{150} \mathrm{Sm}$ & ${ }^{168} \mathrm{Yb}$ & 0.60 & $85 \pm 5$ & $1.85 \pm 0.05$ & $10.4 \pm 2.4$ & $7.3 \pm 2.1$ & & {$[4]$} \\
\hline${ }^{19} \mathrm{~F}+{ }^{159} \mathrm{~Tb}$ & ${ }^{178} \mathrm{~W}$ & 0.64 & $80 \pm 10$ & $1.81 \pm 0.10$ & $10.3 \pm 2.3$ & $7.4 \pm 2.3$ & & {$[5]$} \\
\hline${ }^{19} \mathrm{~F}+{ }^{169} \mathrm{Tm}$ & ${ }^{188} \mathrm{Pt}$ & 0.67 & $80 \pm 5$ & $1.77 \pm 0.05$ & $7.1 \pm 1.2$ & $4.8 \pm 1.1$ & & [5] \\
\hline${ }^{28} \mathrm{Si}+{ }^{170} \mathrm{Er}$ & ${ }^{198} \mathrm{~Pb}$ & 0.70 & $60 \pm 5$ & $1.53 \pm 0.05$ & $7.1 \pm 1.1$ & $5.3 \pm 1.1$ & & {$[4]$} \\
\hline${ }^{19} \mathrm{~F}+{ }^{181} \mathrm{Ta}$ & ${ }^{200} \mathrm{~Pb}$ & 0.70 & $65 \pm 5$ & $1.63 \pm 0.05$ & $8.6 \pm 1.0$ & $6.4 \pm 1.0$ & & [4] \\
\hline${ }^{30} \mathrm{Si}+{ }^{170} \mathrm{Er}$ & ${ }^{200} \mathrm{~Pb}$ & 0.70 & $55 \pm 5$ & $1.43 \pm 0.06$ & $7.0 \pm 0.9$ & $5.5 \pm 0.9$ & & [4] \\
\hline${ }^{18} \mathrm{O}+{ }^{192} \mathrm{Os}$ & ${ }^{210} \mathrm{Po}$ & 0.71 & $60 \pm 5$ & $1.53 \pm 0.05$ & $8.0 \pm 0.8$ & $6.1 \pm 0.8$ & & [4] \\
\hline${ }^{16} \mathrm{O}+{ }^{197} \mathrm{Au}$ & ${ }^{213} \mathrm{Fr}$ & 0.74 & $45 \pm 5$ & $1.33 \pm 0.07$ & $6.2 \pm 0.6$ & $4.8 \pm 0.6$ & & [6] \\
\hline${ }^{16} \mathrm{O}+{ }^{208} \mathrm{~Pb}$ & ${ }^{224} \mathrm{Th}$ & 0.76 & $30 \pm 5$ & $1.08 \pm 0.08$ & $5.5 \pm 0.5$ & $4.7 \pm 0.5$ & & [1] \\
\hline${ }^{19} \mathrm{~F}+{ }^{232} \mathrm{Th}$ & ${ }^{251} \mathrm{Es}$ & 0.83 & $20 \pm 10$ & $0.85 \pm 0.25$ & $1.8 \pm 0.2$ & $1.5 \pm 0.2$ & & [5] \\
\hline$p+{ }^{238} U$ & ${ }^{239} \mathrm{~Np}$ & 0.78 & $20 \pm 2$ & $0.86 \pm 0.05$ & $4.3 \pm 0.1$ & $3.9 \pm 0.1$ & $n$ & {$[8]$} \\
\hline${ }^{28} \mathrm{Si}+{ }^{164} \mathrm{Er}$ & ${ }^{192} \mathrm{~Pb}$ & 0.72 & $58 \pm 5$ & $1.52 \pm 0.06$ & $5.9 \pm 0.9$ & $4.3 \pm 0.8$ & $p$ & [9] \\
\hline${ }^{28} \mathrm{Si}+{ }^{164} \mathrm{Er}$ & ${ }^{192} \mathrm{~Pb}$ & 0.72 & $53 \pm 5$ & $1.47 \pm 0.06$ & $6.7 \pm 0.9$ & $5.1 \pm 0.9$ & $\alpha$ & {$[9]$} \\
\hline${ }^{19} \mathrm{~F}+{ }^{181} \mathrm{Ta}$ & ${ }^{200} \mathrm{~Pb}$ & 0.70 & $68-84$ & $1.66-1.82$ & $8.4-6.5$ & $6.1-4.1$ & $\gamma$ & {$[10]$} \\
\hline${ }^{32} S+{ }^{184} W$ & ${ }^{216} \mathrm{Th}$ & 0.78 & $72-85$ & $1.65-1.77$ & $2.6-1.7$ & $1.4-0.7$ & & {$[11]$} \\
\hline${ }^{16} \mathrm{O}+{ }^{208} \mathrm{~Pb}$ & ${ }^{224} \mathrm{Th}$ & 0.76 & $30-40$ & $1.09-1.22$ & $5.5-4.6$ & $3.7-4.8$ & & {$[12]$} \\
\hline${ }^{32} \mathrm{~S}+{ }^{208} \mathrm{~Pb}$ & ${ }^{240} \mathrm{Cf}$ & 0.84 & $67-80$ & $1.52-1.68$ & $0.7-0.4$ & $0.2-0.1$ & & [13] \\
\hline
\end{tabular}


TABLE II. Decaying nucleus, fissilities $\left(x_{f_{i s s}}\right)$, threshold energies $\left(\mathrm{E}_{t h r e s h}\right)$, threshold temperatures $\left(\mathrm{T}_{\text {thresh }}\right)$, mean fission barriers $\mathrm{E}_{B a r}$, temperature dependent mean fission barriers $\mathrm{E}_{B a r}(\mathrm{~T})$, for the peripheral reaction ${ }^{40} \mathrm{Ar}+{ }^{232} \mathrm{Th}$ from Ref. 14. All energies and temperatures are given in $\mathrm{MeV}$.

\begin{tabular}{lccccc}
\hline \hline Nucleus & $x_{\text {fiss }}$ & $\mathrm{E}_{\text {thresh }}$ & $T_{\text {thresh }}$ & $\mathrm{E}_{\text {Bar }}$ & $\mathrm{E}_{\text {Bar }}(\mathrm{T})$ \\
\hline${ }^{225} \mathrm{Fr}$ & 0.73 & $47 \pm 4$ & $1.29 \pm 0.08$ & $6.0 \pm 0.6$ & $4.8 \pm 0.6$ \\
${ }^{228} \mathrm{Ra}$ & 0.74 & $34 \pm 2$ & $1.07 \pm 0.07$ & $5.3 \pm 0.5$ & $4.6 \pm 0.5$ \\
& 0.74 & $46 \pm 6$ & $1.29 \pm 0.10$ & $5.9 \pm 0.3$ & $4.8 \pm 0.4$ \\
& 0.74 & $66 \pm 7$ & $1.59 \pm 0.10$ & $7.0 \pm 0.2$ & $5.1 \pm 0.4$ \\
${ }^{230} \mathrm{Ac}$ & 0.75 & $18 \pm 2$ & $0.76 \pm 0.12$ & $4.7 \pm 0.4$ & $4.3 \pm 0.5$ \\
& 0.75 & $21 \pm 3$ & $0.82 \pm 0.10$ & $5.2 \pm 0.3$ & $4.8 \pm 0.4$ \\
& 0.75 & $32 \pm 4$ & $1.09 \pm 0.09$ & $6.2 \pm 0.2$ & $5.4 \pm 0.3$ \\
\hline \hline
\end{tabular}




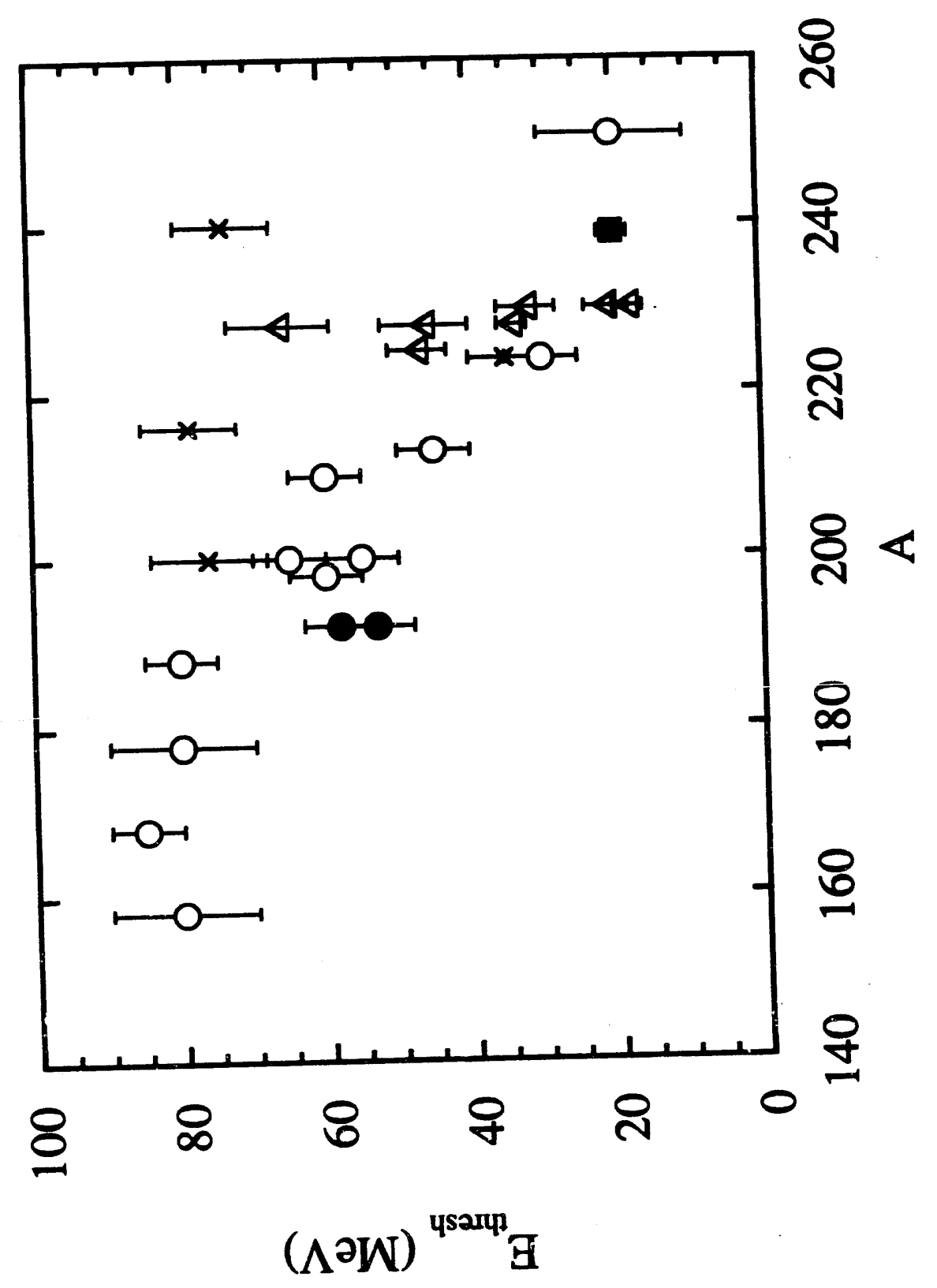




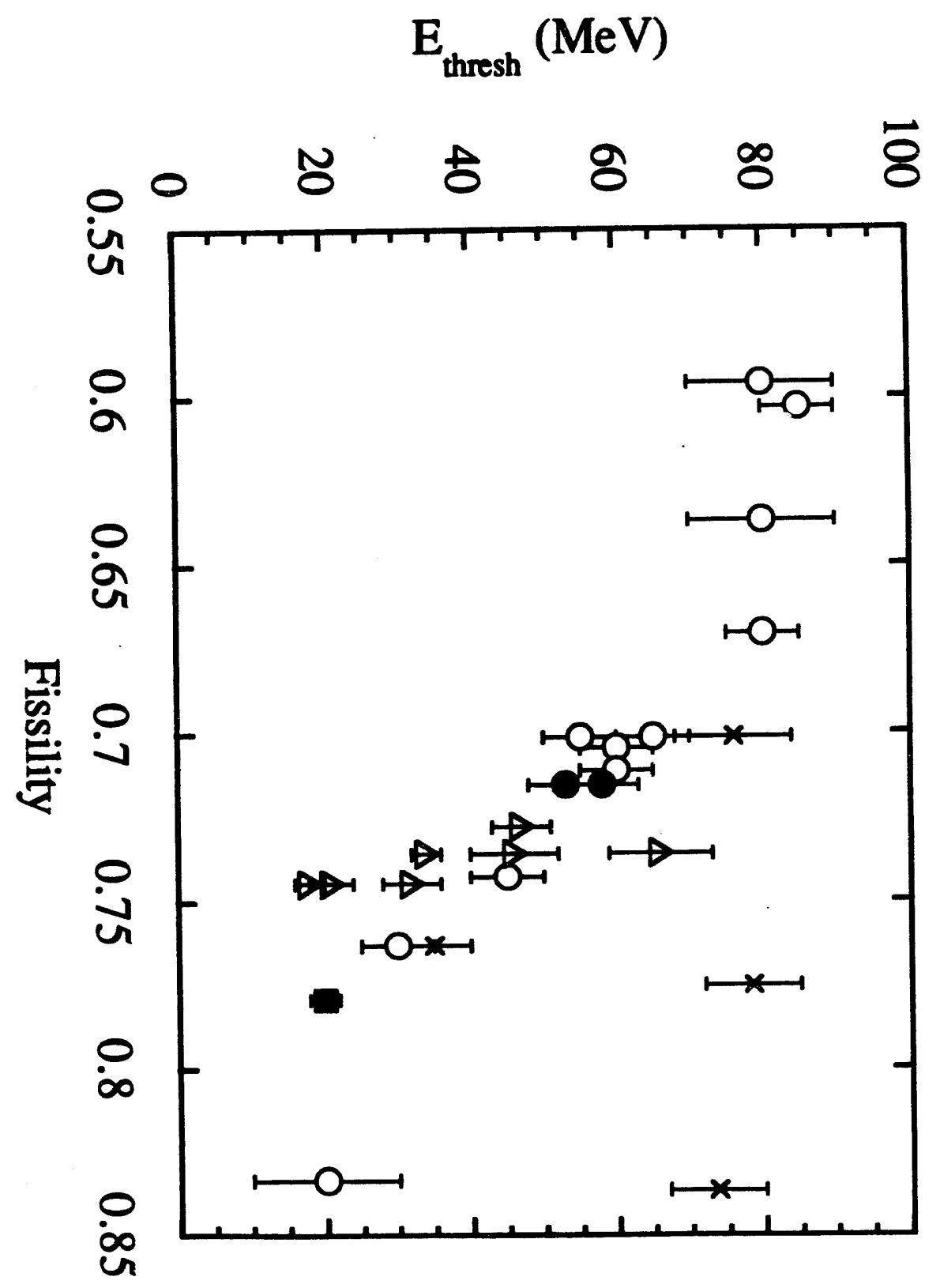




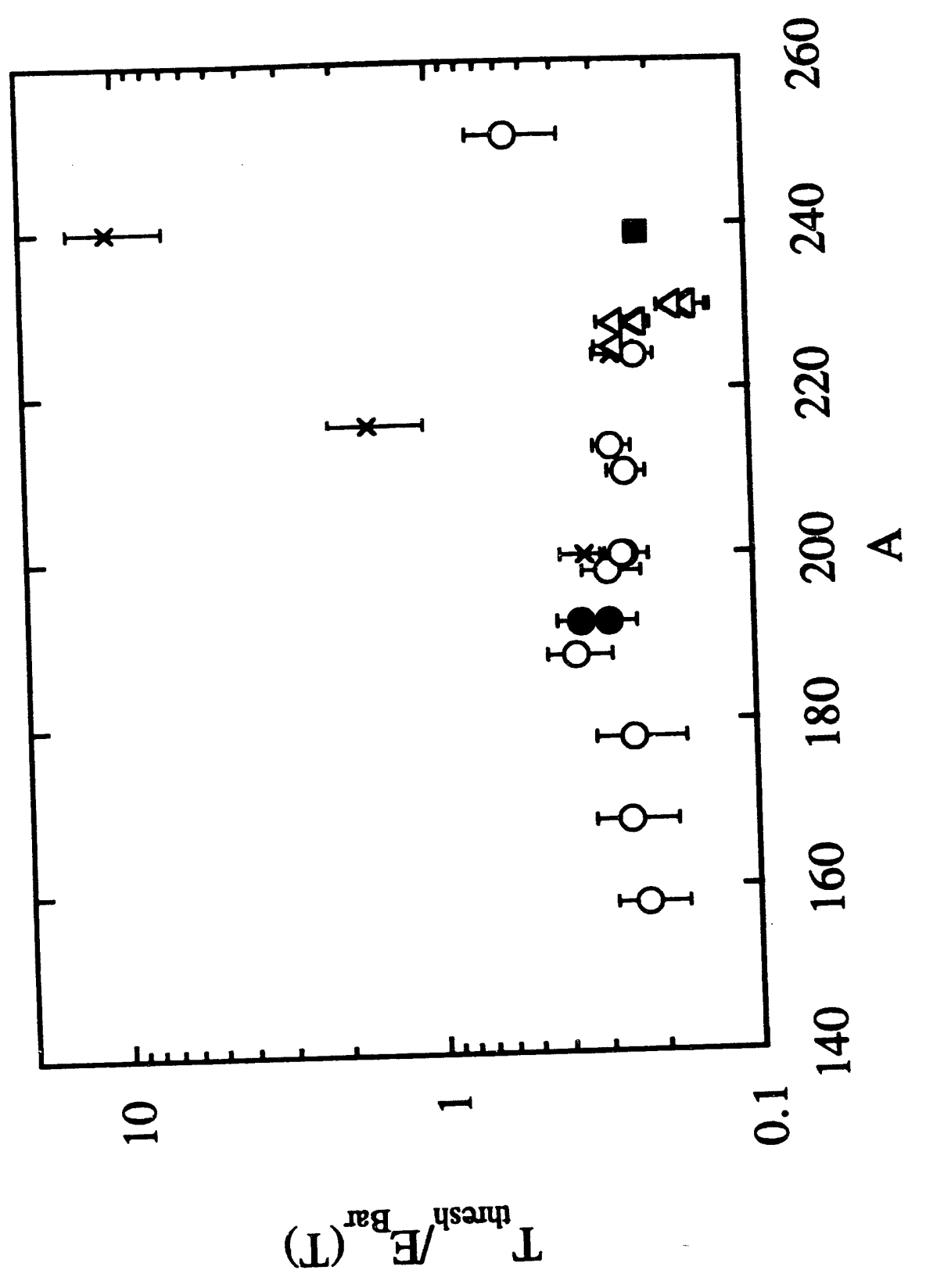




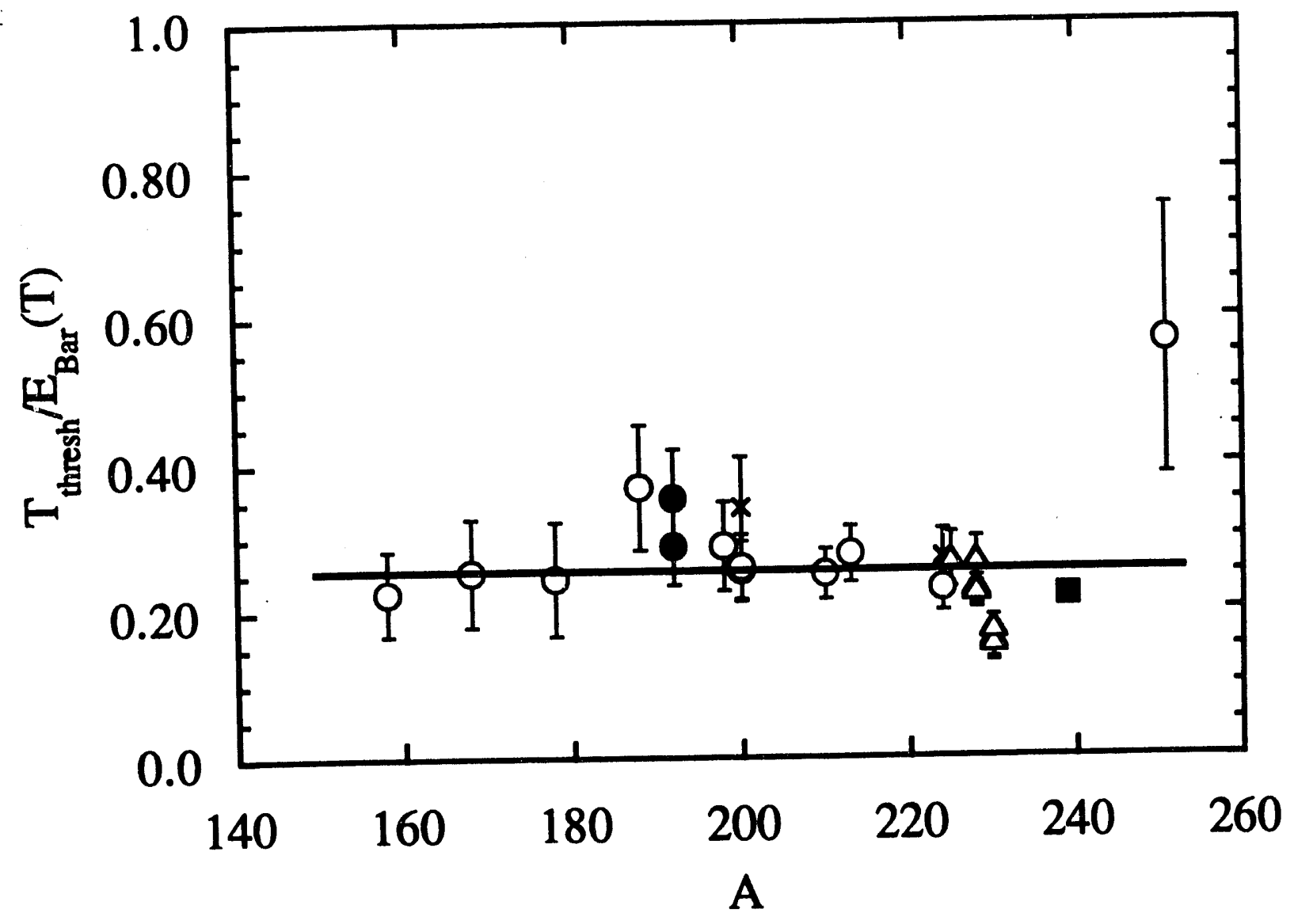

Fil, 

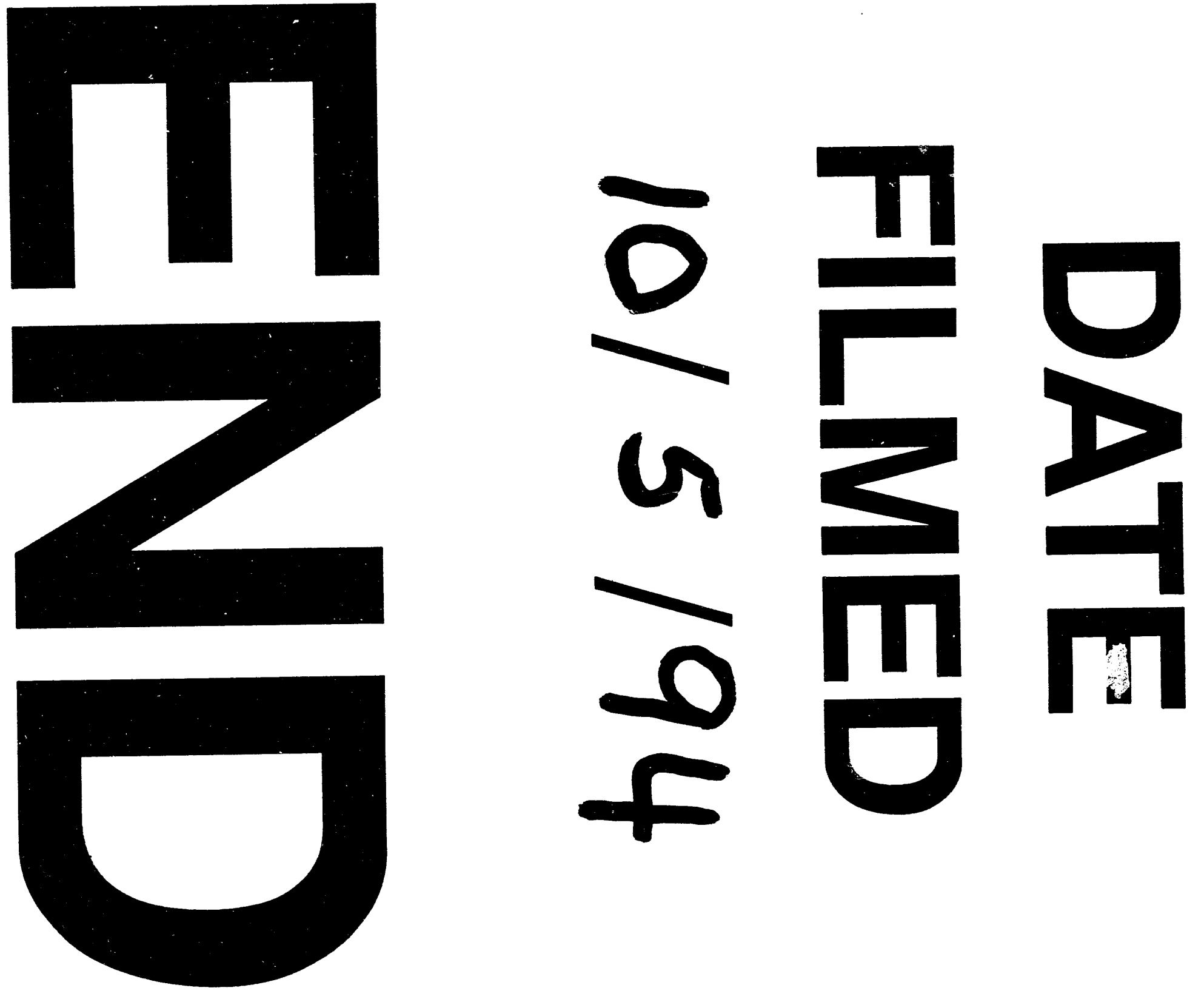
